\title{
Surfactant Mobility in Nanoporous Glass Films
}

\author{
Taek-Soo Kim, ${ }^{\dagger}$ Katherine Mackie, ${ }^{\ddagger}$ Qiping Zhong, ${ }^{\S}$ Maria Peterson, ${ }^{\S}$ \\ Tomohisa Konno, ${ }^{\S}$ and Reinhold H. Dauskardtt, ${ }^{\star, \neq}$ \\ Department of Mechanical Engineering, Stanford University, \\ Stanford, California 94305, Department of Materials Science and Engineering, \\ Stanford University, Stanford, California 94305, and JSR Micro, Inc., 1280 North \\ Mathilda Avenue, Sunnyvale, California 94089
}

Received April 9, 2009; Revised Manuscript Received May 9, 2009

\begin{abstract}
Polymer molecules when physically confined at nanometer length scales diffuse nonclassically and very differently depending on their molecular weight and the nature of the confinement. Long polymers that exhibit "snakelike" reptation based mobility in melts may diffuse faster in confined nanometer sized cylinders with pore diameter $d \sim 15 \mathrm{~nm}$, and short polymers subject to Rouse dynamics have shown signatures of reptation and slower diffusion when confined in nanoporous glass with $d \sim 4 \mathrm{~nm}$. However, the mobility of short polymers with radii of gyration similar to a smaller pore diameter $(d \leq 2.1 \mathrm{~nm})$ but with extended lengths well larger than the pore diameter has not as yet been studied. In this work, we demonstrate that those short molecules including nonionic surfactants can readily diffuse in strongly hydrophobic nanoporous glasses film with $d \leq 2.1 \mathrm{~nm}$. The diffusivity was found sensitive to molecular weight, hydrophilic-lipophilic balance, and molecular structure of surfactants. Remarkably, analysis of the measured diffusion coefficients reveals that short-chain surfactants exhibit signature of reptation based diffusion in the nanoscopic pore confinements. Such reptation mobility in agreement with theoretical predictions is not even observed in reptating polymer melts due to fluctuations of the entanglement pathway. The fixed pathways in the interconnected nanoporous films provide ideal nanoscale environments to explore mobility of confined molecules, and the results have implications for a number of technologies where nanoporous materials are in contact with surfactant molecules.
\end{abstract}

Polymer molecules when physically confined at nanometer length scales diffuse nonclassically and very differently compared to bulk conditions depending on the polymer molecular weight, and the size and shape of the confinement. ${ }^{1-3}$ Long polystyrene molecules with a molecular weight above the entanglement weight $M_{\mathrm{c}} \sim 20 \mathrm{~kg} / \mathrm{mol}$ that exhibit a "snakelike" reptation based mobility in the bulk ${ }^{4}$ were found to diffuse faster in cylindrical alumina nanopores (pore diameter $d \sim 15 \mathrm{~nm}$ ). ${ }^{1}$ The enhanced mobility was related to the lower molecular entanglement associated with fewer chains present in the confined region measured using time-resolved small-angle X-ray scattering (SAXS). On the contrary, short polymers that are subject to Rouse dynamics under bulk conditions ${ }^{5}$ are either reported to exhibit no mobility change in interconnected mesopores ${ }^{6}$ or begin to exhibit some signatures of slower reptation-like motion in nanoporous Vycor glass $(d \sim 4 \mathrm{~nm})$ due to the geometric confinement of the nanometer-sized pores. ${ }^{2}$ The reptationlike motion was characterized by field cycling nuclear magnetic resonance (NMR) relaxometry. These findings

\footnotetext{
* Corresponding author, dauskardt@stanford.edu.

† Department of Mechanical Engineering, Stanford University.

* Department of Materials Science and Engineering, Stanford University.

$\S$ JSR Micro, Inc.
}

involve molecular mobility of pure polymer melts; the short molecule studies involved the dynamics of molecules already contained in the nanoporous glass, and the long molecule study involved molecular mobility associated with flow into the nanoporous media.

The mobility of short polymers with radii of gyration similar to nanoscale pore diameters $(d \leq 2.1 \mathrm{~nm})$ but with extended lengths larger than the pore diameter has not as yet been reported. In fact, studies using nonionic surfactant molecules with these length scales claim that such diffusion does not occur. $^{7-9}$ In this study, we demonstrate that nonionic surfactants can readily diffuse in strongly hydrophobic nanoporous glass film with $d \leq 2.1 \mathrm{~nm}$. The diffusivity was found sensitive to molecular weight, hydrophilic-lipophilic balance, and molecular structure of surfactants. Remarkably, analysis of the measured diffusion coefficients reveals that those short-chain surfactant molecules exhibit a signature of reptation based diffusion in the nanoscopic pore confinements. Our study was conducted using simple optical measurements of the motion of the solution diffusion front. The behavior results in a diffusion constant, $D$, that scales with the molecular weight, $M$, according to $D \sim M^{-2}$, in almost perfect agreement with reptation theory. ${ }^{4}$ The scaling typically observed for reptating polymer melts is $D \sim M^{-2.3}$; 
the discrepancy from theory is generally believed to result from fluctuations of the entanglement pathway that occur in the melt. No such fluctuation occurs in the confined interconnected nanoporous films, and the theoretically predicted scaling was therefore observed.

The mobility of such molecules in the geometrically highly confined interconnected nanoporous regions presents interesting fundamental and technological questions. Nanoporous films are being actively developed for use as microelectronic devices, ${ }^{10-15}$ size-selective membranes,${ }^{16}$ biosensors, ${ }^{17}$ optical waveguides, ${ }^{18,19}$ and photovoltaic cells. ${ }^{20}$ The pores in nanoporous materials containing more than $\sim 20$ vol \% porosity are nearly always interconnected, and the films are frequently exposed to molecular species in process or service environments. In particular, molecular diffusion is not always anticipated when the pore diameter approaches the size of the surfactant molecule, and a paucity of data and the lack of understanding are compounded by the experimental complexity of characterizing molecular diffusion in thin nanoporous films.

We further report on the effects of molecular structure including linear and branched surfactants and the hydrophiliclipophilic balance (HLB) on molecular mobility in the films. The critical role of the water present was examined in terms of modifications to the polar interactions between the molecules and between the molecules and the pore surfaces. The molecules selected are types of nonionic surfactants ubiquitously employed in a wide range of technologies. For example, they are essential components of slurries for chemical mechanical planarization (CMP) of emerging classes of nanoporous glass films where they are vital for enhanced wetting, removal rates, slurry stability, and selectivity. In the case of ultralow dielectric constant nanoporous films, molecular-assisted diffusion of such CMP slurries into the films can have dramatic effects on the resulting dielectric constant. ${ }^{7-9,21,22}$ The behavior of surfactant molecules in these films is not well understood and the lack of the knowledge has generated the misconception that surfactants do not penetrate the films but adsorb on the film surface and simply assist water diffusion into the pores. ${ }^{7-9}$

A wide range of nonionic surfactants including monomeric (linear bridged) polyoxyethylene alkyl ethers and dimeric (branched) gemini surfactants were selected for study (Figure 1). Both 0.1 wt $\%$ aqueous surfactant solutions ( $\mathrm{pH} 7$ deionized water adjusted by $\mathrm{NH}_{4} \mathrm{OH}$ ) and 100 wt \% surfactants in liquid phase at lab air temperature $\sim 23 \pm 2$ ${ }^{\circ} \mathrm{C}$ were tested for diffusion using the molecules listed in Table 1. Polyoxyethylene alkyl ethers, $\mathrm{C}_{m} \mathrm{E}_{n}$, with various hydrophobic alkyl tail lengths, $m$, and hydrophilic ethylene oxide (EO) headgroup lengths, $n$, were selected to study systematically the roles of the hydrophobic and hydrophilic group lengths on the surfactant diffusion in nanoporous films. Dimeric surfactants were selected to reveal the role of the molecular structure of surfactants in the diffusion. The hydrophilic-lipophilic balance (HLB) of the surfactants was calculated by Griffin's method: ${ }^{37} \mathrm{HLB}=20 M_{\mathrm{h}} / M$, where $M_{\mathrm{h}}$ is the molecular mass of the hydrophilic portion of the molecule and $M$ is the molecular mass of the whole molecule. (a)

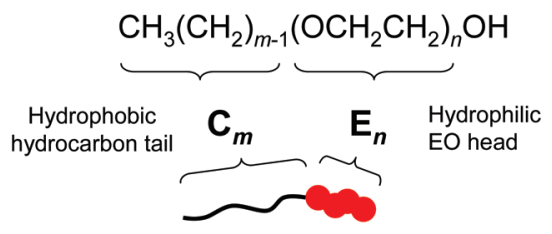

(b)

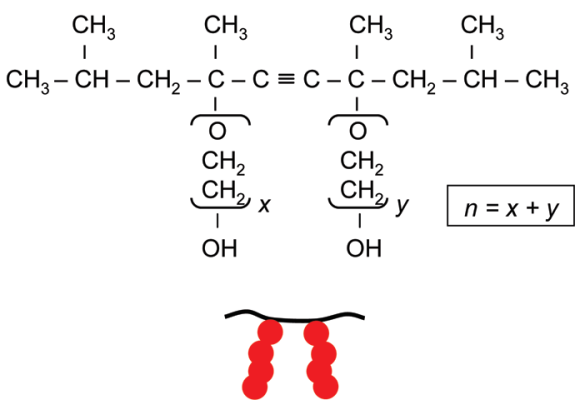

Figure 1. Molecular structure of (a) polyoxyethylene alkyl ethers and (b) dimeric nonionic surfactants.

The lateral solvent diffusion technique ${ }^{21-27}$ was adopted to characterize the diffusion of surfactants in liquid phase and in aqueous solution (Figure 2a). Nanoporous methylsilsesquioxane (MSSQ) thin films were deposited on silicon substrates using spin-on techniques and cured by broad band UV irradiation. The final film thickness was $500 \mathrm{~nm}$ and had a density $\rho \sim 1.06 \mathrm{~g} / \mathrm{cm}^{3}$ and pore diameter $d \sim 2.1 \mathrm{~nm}$ measured by positron annihilation lifetime spectroscopy (PALS), and a dielectric constant $k \sim 2.3$. The MSSQ glass matrix is a structurally modified form of silica produced by replacing a bridging oxygen atom with a terminal methyl group yielding a film with reduced $k$ and strong hydrophobicity. The top surface of the film was hermetically sealed with an optically transparent $\operatorname{SiN}_{x}$ cap. The capping layer was deposited by PECVD in two consecutive steps to prevent the formations of pinholes in the layer by first depositing $100 \mathrm{~nm}$ of $\mathrm{SiN}_{x}$ and then rotating the wafer by $90^{\circ}$ and depositing a second $100 \mathrm{~nm}$ of $\mathrm{SiN}_{x}$. The silicon substrates containing the films were cleaved to expose a fresh throughthickness surface of the film and substrate and immersed in the solution of interest. Diffusion of the solution into the plane of the film from the freshly exposed film surface resulted in a change in the refractive index (RI) and color of the film that could be easily monitored by optical observation through the top surface of the films using an optical microscope. Diffusion of the surfactant solution into the nanoporous films was clearly apparent as shown in the optical micrograph in Figure $2 \mathrm{~b}$ and was monitored as a function of time. The $\mathrm{pH} 7$ aqueous solutions with $0.1 \mathrm{wt} \%$ $\mathrm{C}_{m} \mathrm{E}_{n}$ surfactants diffused rapidly into the nanoporous glass films over the time periods examined as shown in Figure 3 where diffusion distance, $x$, is plotted in terms of square root of diffusion time, $t^{0.5}$. However, the same $\mathrm{pH} 7$ aqueous solutions without surfactants exhibited no detectable diffusion. The linear relationship observed in the resulting plots is characteristic of one-dimensional Fickian diffusion with diffusion distance given by $x=(D t)^{0.5}$ where $D$ is the diffusion coefficient. ${ }^{22}$ The diffusion coefficient was calcu- 
Table 1. Polyoxyethylene Alkyl Ethers and Dimeric Surfactants Selected for the Diffusion Tests and Their Measured Diffusion Coefficients

\begin{tabular}{|c|c|c|c|c|c|c|}
\hline name & $\begin{array}{l}\text { no. of } \\
\mathrm{C}, m\end{array}$ & $\begin{array}{l}\text { no. of } \\
\mathrm{EO}, n\end{array}$ & $\begin{array}{c}\text { hydrophilic-lipophilic } \\
\text { balance, HLB }\end{array}$ & $\begin{array}{l}\text { molecular weight, } \\
M(\mathrm{~g} / \mathrm{mol})\end{array}$ & $\begin{array}{c}0.1 \text { wt } \% \text { surfactant } \\
\text { diffusion coefficient, } D \\
\left(\mathrm{~m}^{2} \mathrm{~s}^{-1}\right)\end{array}$ & $\begin{array}{l}100 \text { wt } \% \text { surfactant } \\
\text { diffusion coefficient, } D \\
\left(\mathrm{~m}^{2} \mathrm{~s}^{-1}\right)\end{array}$ \\
\hline $\mathrm{C}_{10} \mathrm{E}_{4}$ & 10 & 4 & 10.5 & 334 & $2.77 \times 10^{-12}$ & $2.72 \times 10^{-12}$ \\
\hline $\mathrm{C}_{10} \mathrm{E}_{6}$ & & 6 & 12.4 & 423 & $2.19 \times 10^{-12}$ & $1.99 \times 10^{-12}$ \\
\hline $\mathrm{C}_{10} \mathrm{E}_{9}$ & & 9 & 14.3 & 555 & $1.14 \times 10^{-12}$ & $1.44 \times 10^{-12}$ \\
\hline $\mathrm{C}_{12} \mathrm{E}_{4}$ & 12 & 4 & 9.2 & 363 & $3.12 \times 10^{-12}$ & $7.67 \times 10^{-12}$ \\
\hline $\mathrm{C}_{12} \mathrm{E}_{7}$ & & 7 & 12.2 & 495 & $2.4 \times 10^{-12}$ & $5.11 \times 10^{-12}$ \\
\hline $\mathrm{C}_{12} \mathrm{E}_{23}$ & & 23 & 16.8 & 1200 & $1.9 \times 10^{-13}$ & \\
\hline $\mathrm{C}_{12} \mathrm{E}_{50}$ & & 50 & 18.3 & 2389 & $7.8 \times 10^{-14}$ & \\
\hline $\mathrm{C}_{18} \mathrm{E}_{10}$ & 18 & 10 & 12.4 & 711 & $1.45 \times 10^{-13}$ & \\
\hline $\mathrm{C}_{18} \mathrm{E}_{20}$ & & 20 & 15.3 & 1,152 & $1.18 \times 10^{-13}$ & \\
\hline $\mathrm{C}_{18} \mathrm{E}_{100}$ & & 100 & 18.8 & 4676 & $3.28 \times 10^{-14}$ & \\
\hline D-1 & 14 & 1.3 & 4 & 284 & $2.06 \times 10^{-13}$ & $8.20 \times 10^{-14}$ \\
\hline D-2 & & 3.5 & 8 & 381 & $7.29 \times 10^{-14}$ & $7.84 \times 10^{-14}$ \\
\hline D-3 & & 10 & 13 & 667 & $7.29 \times 10^{-14}$ & $7.32 \times 10^{-14}$ \\
\hline D-4 & & 30 & 17 & 1548 & $7.47 \times 10^{-15}$ & $6.45 \times 10^{-14}$ \\
\hline
\end{tabular}

lated from the slope of a linear fit of the diffusion distance as a function of the square root of time and values are listed in Table 1. These were found to decrease with increasing molecular weight, $M$, and for a given alkyl tail length, $m$, with increasing $\mathrm{EO}$ headgroup length, $n$ (i.e., increasing HLB).

The observed diffusion of the aqueous solutions does not necessarily mean that the surfactant molecules themselves penetrate into the nanoporous films. The $0.1 \mathrm{wt} \%$ solutions are well above the critical micelle concentration $(\mathrm{cmc})$ suggesting that the surfactant molecules are contained in large micellular assemblies that would be sterically prevented from diffusing in the interconnected nanopores. In addition, the extended linear lengths of the molecules themselves are 2.9 $\mathrm{nm}\left(\mathrm{C}_{10} \mathrm{E}_{4}\right)$ to $37.9 \mathrm{~nm}\left(\mathrm{C}_{18} \mathrm{E}_{100}\right)$ and far larger than the pore diameter of $\sim 2.1 \mathrm{~nm}$. For these reasons it has been assumed that the surfactant's only role may be to adsorb on the surface of the film and assist water molecule diffusion into the pores. $^{7-9}$

To demonstrate that the surfactant molecules do in fact diffuse into the films, we examined the diffusion of pure surfactants. Diffusion fronts were clearly apparent for the pure surfactants, and the measured diffusion coefficients were similar or even greater than those of the $0.1 \mathrm{wt} \%$ surfactant aqueous solutions (Table 1). This clearly demonstrated the ability of the surfactants to penetrate into the highly confined
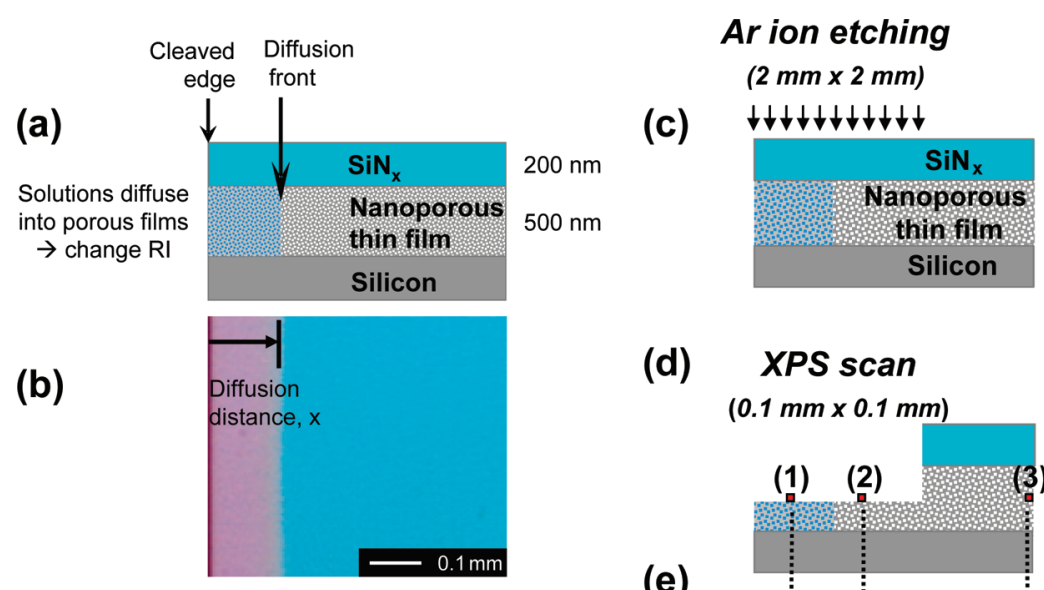

\section{(d) XPS scan}

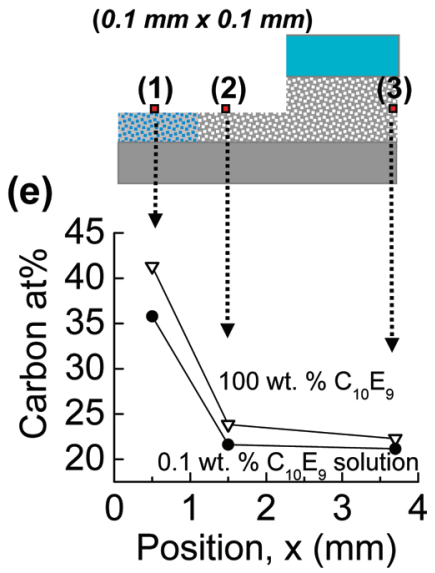

Figure 2. Lateral solvent diffusion technique adopted to measure the diffusion of surfactants through the hydrophobic nanoporous thin film: (a) schematic diagram of the cross-sectional view of a test specimen; (b) an optical image showing the change in color due to the diffusion of solutions into the films as a result of the change in refractive index (top down view); (c) following the diffusion tests, a square etch crater was made all the way down to the middle of the nanoporous film thickness, (d) then several selected regions on the crater were scanned by XPS and (e) the presence of surfactants in the nanoporous films were probed by tracing the increase of carbon content along the diffusion axis in the nanoporous films. 
(a)

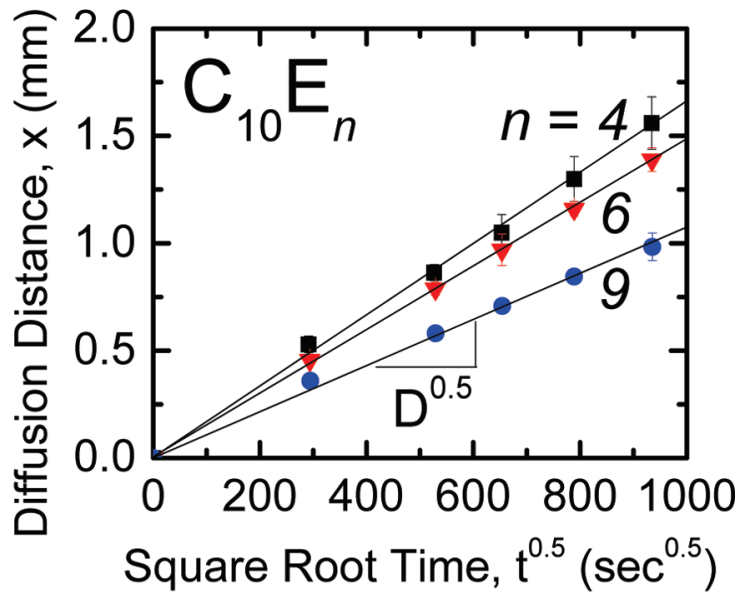

(b)

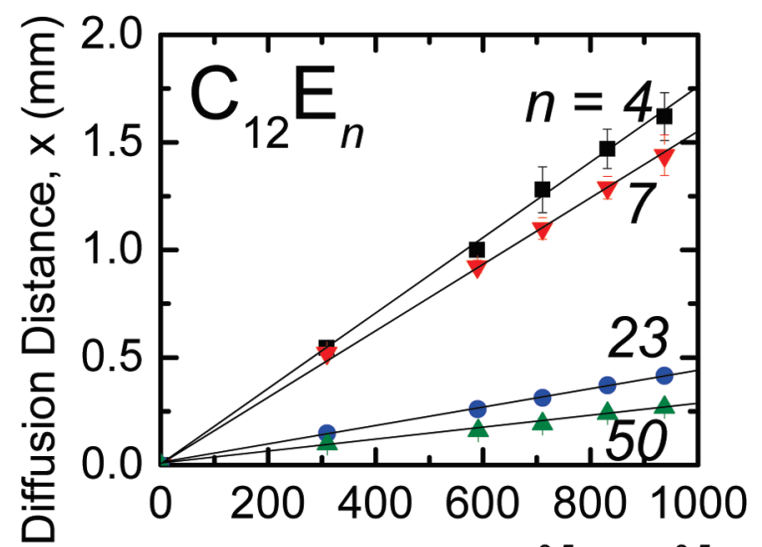

Square Root Time, $\mathrm{t}^{0.5}\left(\mathrm{sec}^{0.5}\right)$

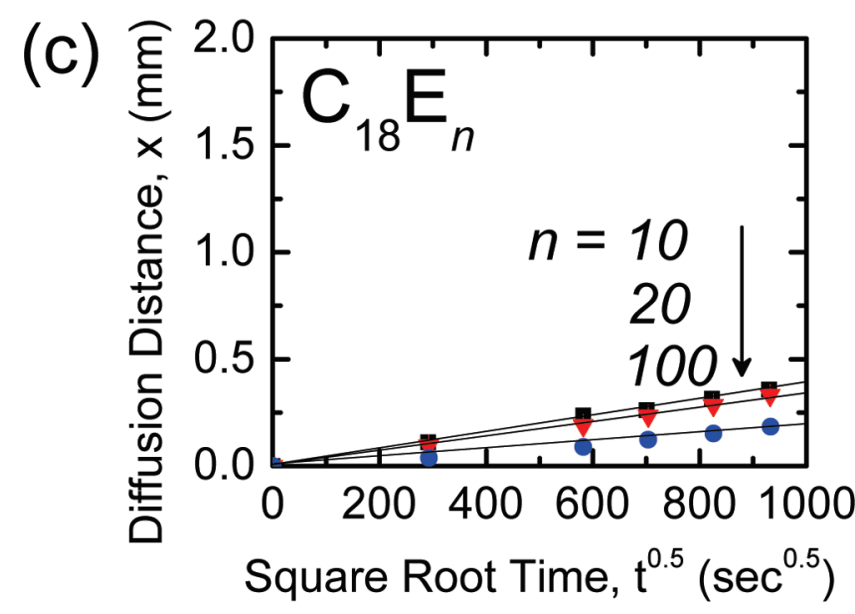

Figure 3. Diffusion distance in the nanoporous MSSQ as a function of square root time of $\mathrm{pH} 7$ aqueous solutions containing $0.1 \mathrm{wt} \%$ of (a) $\mathrm{C}_{10} \mathrm{E}_{n}$, (b) $\mathrm{C}_{12} \mathrm{E}_{n}$, and (c) $\mathrm{C}_{18} \mathrm{E}_{n}$ surfactants, respectively.

nanoporous regions and further suggested that the diffusion of the surfactant molecules in the aqueous solutions was not the rate-limiting step for the observed slower diffusion of those solutions. The diffusion coefficients of the pure surfactants decreased with increasing EO proportion of the molecular weight and consequently increasing HLB of the surfactants. The pure $\mathrm{C}_{12} \mathrm{E}_{23}, \mathrm{C}_{12} \mathrm{E}_{50}, \mathrm{C}_{18} \mathrm{E}_{10}, \mathrm{C}_{18} \mathrm{E}_{20}$, and $\mathrm{C}_{18} \mathrm{E}_{100}$ surfactants were not studied because they were in solid phase at room temperature.
Following diffusion, the presence of surfactants in the nanoporous films was directly confirmed by measuring the increase in carbon content along the diffusion direction in the nanoporous films using X-ray photoelectron spectroscopy (XPS) (Figure $2 \mathrm{c}-\mathrm{e}$ ). The carbon content in the diffusion region labeled position (1) was $\sim 41 \%$ for the pure $\mathrm{C}_{10} \mathrm{E}_{9}$ surfactant and $\sim 36 \%$ for the 0.1 wt $\% \mathrm{C}_{10} \mathrm{E}_{9}$ solution and dropped significantly at position (2) directly ahead of the optically observed diffusion front. At position (3) well ahead of the diffusion front, the carbon content was $\sim 20$ atom $\%$, indicative of the expected carbon content of the pristine nanoporous methylsilsesquioxane (MSSQ) film. The large increase of the carbon content confirms the presence of the surfactant molecules. A surprising observation was that the measured carbon content after diffusion of the dilute 0.1 wt $\%$ surfactant solution was not much reduced from that observed after diffusion of the pure surfactant. This suggests again that the diffusion of the surfactant solutions is controlled mainly by the mobility of surfactant molecules rather than the water molecules in solution.

In order to examine the effects of even greater physical confinement, we found that the surfactants were even able to diffuse in an organosilicate glass film deposited by PECVD with a much smaller pore diameter of $0.6 \mathrm{~nm}$. Its density $\rho$ and dielectric constant $k$ were $1.20 \mathrm{~g} / \mathrm{cm}^{3}$ and 2.7 , respectively. The measured $D$ was $4.0 \times 10^{-14}$ and $2.3 \times$ $10^{-14} \mathrm{~m}^{2} \mathrm{~s}^{-1}$ for the pure surfactant and 0.1 wt $\% \mathrm{C}_{10} \mathrm{E}_{4}$ surfactant solution, respectively. While the increased confinement clearly reduced the diffusion rates, surfactant mobility in the interconnected pores was nevertheless clearly apparent and the measured diffusion coefficients of the pure surfactants were again larger than those of the 0.1 wt $\%$ surfactant solutions.

The diffusion of dimeric surfactants was also characterized and compared with the results of $\mathrm{C}_{m} \mathrm{E}_{n}$ surfactants to study the role of the surfactant molecular structures on diffusion. The main structural difference of the two is that $\mathrm{C}_{m} \mathrm{E}_{n}$ surfactants are monomeric (linear) and the dimeric surfactants are branched. The dimeric surfactants are far more mobile in solution and also more surface active because they contain two hydrophiles within a single molecule compared to the single hydrophile/hydrophobe structure of the $\mathrm{C}_{m} \mathrm{E}_{n}$ surfactants. However, for similar molecular weight the diffusion coefficients of the dimeric surfactants were found to be significantly lower than those of the $\mathrm{C}_{m} \mathrm{E}_{n}$ surfactants in the nanoporous MSSQ films. The measured diffusion coefficients are listed in Table 1 and shown as a function of $M$ on a $\log -\log$ plot in Figure 4a,b.

The nanometer scale confinement reduced the surfactant molecular mobility and diffusion coefficients in the nanoporous MSSQ by approximately 2 orders of magnitude from reported values of $4.3 \times 10^{-10} \mathrm{~m}^{2} \mathrm{~s}^{-1}$ for $\mathrm{C}_{10} \mathrm{E}_{8}$ and $4.8 \times$ $10^{-10} \mathrm{~m}^{2} \mathrm{~s}^{-1}$ for $\mathrm{C}_{12} \mathrm{E}_{8}$ measured in the bulk melts or solutions. ${ }^{28,29}$ Moreover, the diffusion coefficients of the surfactant solutions in the nanoporous MSSQ are nearly proportional to $M^{-2}$ as shown in Figure 4a. This coincides with the scaling-law predicted for molecular reptation where $D \sim M^{-2}$ which was proposed by de Gennes ${ }^{4}$ to explain the 
(a)

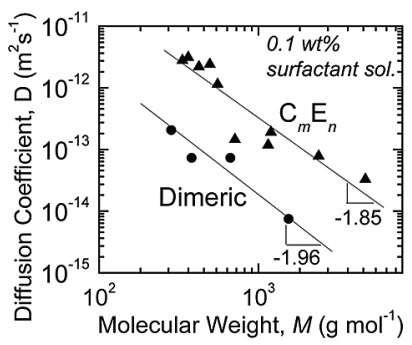

(b)

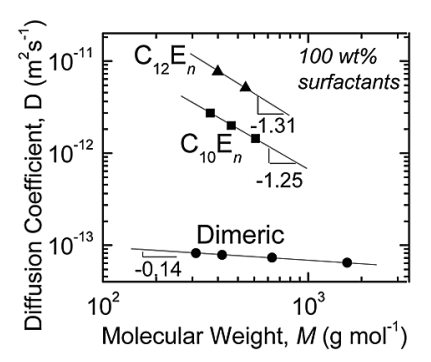

(c)

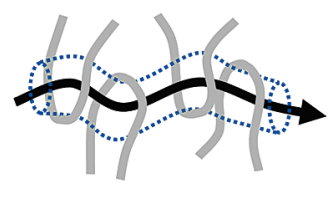

(d)

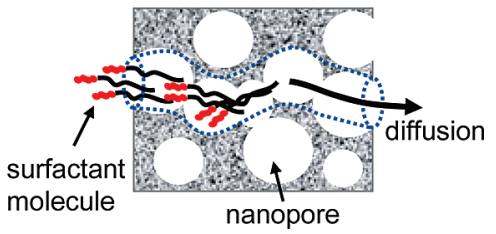

Figure 4. Diffusion coefficients in terms of $M$ in $\log -\log$ scale for (a) $0.1 \mathrm{wt} \%$ surfactant solutions and (b) $100 \mathrm{wt} \%$ surfactants. Slopes were calculated from the linear fits. Schematic diagrams of diffusion by reptation: (c) the classical reptation model regards a polymer chain (a black solid line) in an entangled melt or concentrated solution as being within a virtual tube (dotted lines) defined by the locus of its intersections with other melt molecules (gray solid lines), and (d) short-chain surfactants diffuse through an real tube formed by interconnected nanopores in nanoporous glass.

motion of a polymer molecule in a concentrated (or molten) polymer network. According to the reptation model the motion of polymer molecules above a critical entanglement length are confined within virtual tubes that are defined by the locus of intersections (or points of entanglement) with adjacent molecules (Figure 4c).$^{30}$ The reptation model has been confirmed experimentally in polymer melts by infrared microdensitometry, ${ }^{30}$ forced Rayleigh scattering, ${ }^{31}$ and field gradient $\mathrm{NMR},{ }^{32,33}$ although the scaling typically observed for reptating polymer melts is $D \sim M^{-2.3}$, and the discrepancy from the theoretical scaling of $D \sim M^{-2}$ is believed to result from fluctuations of the entanglement pathway that occur in the melt.

The motion of short polymers similar to the surfactants used in the present study that are well below the entanglement molecular weight do not exhibit reptation mobility as the polymers are not long enough to provide entanglement confinement. However, instead of the fictitious tube anticipated in the reptation model (Figure 4c) the short-chain surfactant molecules in the nanoporous glass films are physically confined in real tubes formed by interconnected nanometer-sized pores (Figure 4d). Since the tubes formed in the nanoporous glass films do not fluctuate, the $D \sim M^{-2}$ scaling we observe in our experiments is almost in perfect agreement with reptation theory. Note that the estimated diameter of the fictitious tubes (entanglement distance) in polymer melts is generally several nanometers ${ }^{34}$ and is similar to the nanopore diamter $d \sim 2.1 \mathrm{~nm}$. Note also that estimates of the radius of gyration of the surfactants ranges from 0.7 $\mathrm{nm}\left(\mathrm{C}_{10} \mathrm{E}_{4}\right)$ to $2.5 \mathrm{~nm}\left(\mathrm{C}_{18} \mathrm{E}_{100}\right)$ which means that the molecules could nearly fit into the nanopores but would then not exhibit reptation scaling. They must, therefore, become

extended to dimensions closer to their linear lengths (2.9 $\mathrm{nm}\left(\mathrm{C}_{10} \mathrm{E}_{4}\right)$ to $\left.37.9 \mathrm{~nm}\left(\mathrm{C}_{18} \mathrm{E}_{100}\right)\right)$ which would involve a significant loss of conformational entropy when entering the confined nanoporous regions in order to exhibit reptation mobility.

The significantly lower diffusion coefficients of the dimeric surfactants (Figure 4a,b) are also consistent with the reptation theory of a branched chain: when a reptating chain is branched or has some long side groups, its motions are strongly quenched. ${ }^{35}$ Other recent studies using field cycling NMR relaxometry have also revealed that relatively short chain polymers show signatures of reptation when confined in nanoporous media, ${ }^{2,3}$ although the combination of larger pore sizes and molecular weight did not reveal the precise scaling of the present study.

A further intriguing finding was that the diffusion coefficients of the pure surfactants did not scale with $M^{-2}$ (Figure $4 \mathrm{~b}$ ) but exhibited a reduced scaling of $\sim M^{-1.3}$. This raises the question of what makes the surfactant molecules from pure melts diffuse so differently in the nanoporous glasses compared to those from dilute aqueous solutions. While the surfactants were dilute $(0.1 \mathrm{wt} \%)$ in solution, the actual concentration in the nanopores was high and comparable to that of the pure surfactants as revealed by XPS (Figure 2e). The explanation may be related to the role of water in significantly modifying the polar interactions between the surfactant headgroups and interactions of the surfactant molecules with the pore surfaces. The effects of molecular interactions with each other and with surfaces are not included in the original reptation model and are important when molecules are physically confined or near interacting surfaces. The modified reptation dynamics for a polymer melt near an attractive solid surface that accounts for increased molecular friction due to surface-molecular interactions reveal a reduced scaling relation of $D \sim M^{-1.5}$ that depends on the type of interaction. ${ }^{36}$

In aqueous solutions, water molecules may have two effects that explain the behavior. They are strongly attracted to the hydrophilic EO group of the surfactant molecule and on surrounding the group essentially to shield it from polar interactions with other EO groups or with hydroxide groups on the pore surfaces. In addition, water molecules may interact directly with hydroxide groups on the pore surfaces, again decreasing the polar interactions. In both cases we speculate that in aqueous solutions the water molecules hinder the interaction (reduce the friction) of the surfactant molecules with each other and with the confining pore surfaces, providing low friction pathways in which the molecules reptate in almost perfect agreement with reptation theory. On the other hand, in the absence of water, diffusion is retarded and modified by molecular interactions and the diffusion coefficients of the $\mathrm{C}_{m} \mathrm{E}_{n}$ surfactant melts scales with $M^{-1.3}$ in closer agreement with modified reptation dynamics. This may also explain why the surfactants with a higher HLB diffused slower in the nanoporous glass films due to the higher friction between the EO chains and the pore surfaces. The diffusion coefficients of the $\mathrm{C}_{10} \mathrm{E}_{n}$ surfactants were smaller than those of the $\mathrm{C}_{12} \mathrm{E}_{n}$ surfactants for a given $M$ 
(Figure $4 \mathrm{~b}$ ) because the HLB of the $\mathrm{C}_{10} \mathrm{E}_{n}$ was higher than that of the $\mathrm{C}_{12} \mathrm{E}_{n}$.

We note finally that the surface roughness of the interfaces adjacent to the MSSQ layer is unlikely to affect the reported diffusion kinetics since the rms roughness values measured with atomic force microscopy $(\sim 1.5 \mathrm{~nm}$ on the top surface of the MSSQ and $<1 \mathrm{~nm}$ on the $\mathrm{Si}$ ) were negligible compared to the nanoporous film thickness $(500 \mathrm{~nm})$. Another possible influence may have involved hydrophobic/hydrophilic interactions between permeating molecules and the surfaces of $\mathrm{SiN}_{x}$ and $\mathrm{Si}$. We believe the interactions are negligible for several reasons. First, there are very thin dense skin layers $(<5 \mathrm{~nm})$ at the top and bottom of the MSSQ film and no open pores in direct contact with the adjacent layers. This will shield hydrophobic/hydrophilic interactions of the $\operatorname{SiN}_{x}$ and $\mathrm{Si}$ on the diffusion kinetics. Second, the MSSQ is itself strongly hydrophobic (contact angle $>100^{\circ}$ ) and would dominate through the thickness of the film effects of hydrophobicity of the interfaces. Finally, the actual length scale of the hydrophobic/hydrophilic interactions would be small compared to the thickness of the MSSQ film.

We demonstrated that the common nonionic surfactant molecules with extended length above the pore diameter in aqueous solutions or from pure melts diffuse readily into strongly hydrophobic films with pore diameters of $\sim 2.1 \mathrm{~nm}$ and even in films with a much smaller pore diameter $\sim 0.6$ $\mathrm{nm}$. No diffusion was observed for the aqueous solutions without surfactants confirming that the diffusion process was controlled by the diffusion of the surfactant molecules into the nanoporous films. The diffusivity was sensitive to molecular weight, HLB, and molecular structure of linear and branched surfactants. Remarkably, the surfactants exhibited mobility in interconnected nanoporous glass films that is in almost perfect agreement with the scaling predicted by reptation theory, even though the extended lengths of the surfactants are well below the entanglement molecular size needed for reptation-based mobility in melts. The reduced mobility of surfactant molecules from pure melts was explained in terms of the increased molecular interactions that result in the absence of water molecules that shield the molecular headgroups and pore surfaces from polar interactions providing a frictionless pathway for reptation. While this is not complete proof of reptation of the organic molecules under nanoscale confinements, this study provides a signature of the reptation for additional research investigation using field cycling NMR and SAXS.

Acknowledgment. This work was supported by the Director, Office of Energy Research, Office of Basic Energy Sciences, Materials Sciences Division of the U.S. Department of Energy, under Contract No. DE-FG02-07ER46391. T. Kim was supported in part by JSR Micro, Inc.

\section{References}

(1) Shin, K.; Obukhov, S.; Chen, J.; Hun, J.; Hwang, Y.; Mok, S.; Dobriyal, P.; Thiyagarajan, P.; Russell, T. Nat. Mater. 2007, 6, 961965 .
(2) Kausik, R.; Fatkullin, N.; Hqsing, N.; Kimmich, R. Magn. Reson. Imaging 2007, 25, 489-492.

(3) Fatkullin, N.; Kausik, R.; Kimmich, R. J. Chem. Phys. 2007, 126, 094904.

(4) de Gennes, P. G. J. Chem. Phys. 1971, 55 (2), 572-579.

(5) Kausik, R.; Mattea, C.; Fatkullin, N.; Kimmich, R. J. Chem. Phys. 2006, $124,114903$.

(6) Huber, P.; Grüner, S.; Schäfer, C.; Knorr, K.; Kityk, A. V. Eur. Phys. J. Spec. Top. 2007, 141, 101-105.

(7) Kondo, S.; Fukaya, K.; Ohashi, N.; Miyazaki, T.; Nagano, H.; Wada, Y.; Ishibashi, T.; Kato, M.; Yoneda, K.; Soda, E.; Nakao, S.; Ishigami, K.; Kobayashi, N. Proc. IEEE Int. Interconnect Technol. Conf. 2006, $164-166$.

(8) Kondo, S.; Shiohara, M.; Maruyama, K.; Fukaya, K.; Yamada, K.; Ogawa, S.; Saito, S. Proc. IEEE Int. Interconnect Technol. Conf. 2007, $172-174$.

(9) Kondo, S.; Fukaya, K.; Yamada, K.; Miyazaki, T.; Fujita, M.; Abe, D.; Kunisaki, S.; Enomoto, T.; Tominaga, S. Microelectron. Eng. 2007, $84,2615-2619$.

(10) International Technology Roadmap for Semiconductors, 2007 Edition, Interconnect; Semiconductor Industry Association: San Jose, CA, 2007; p 38.

(11) Guyer, E. P.; Dauskardt, R. H. Nat. Mater. 2004, 3 (1), 53-57.

(12) Maidenberg, D. A.; Volksen, W.; Miller, R. D.; Dauskardt, R. H. Nat. Mater. 2004, 3, 464-469.

(13) Guyer, E. P.; Patz, M.; Dauskardt, R. H. J. Mater. Res. 2006, 21 (4), 882-894.

(14) Kim, T.; Tsuji, N.; Kemeling, N.; Matsushita, K.; Chumakov, D.; Geisler, H.; Zschech, E.; Dauskardt, R. H. J. Appl. Phys. 2008, 103, 064108.

(15) Kim, T.; Tsuji, N.; Matsushita, K.; Kobayashi, N.; Chumakov, D.; Geisler, H.; Zschech, E.; Dauskardt, R. H. J. Appl. Phys. 2008, 104, 074113.

(16) Cheetham, A. K.; Ferey, G.; Loiseau, T. Angew. Chem., Int. Ed. 1999, 38, 3268-3292.

(17) Desai, T. A.; Hansford, D. J.; Leoni, L.; Essenpreis, M.; Ferrari, M. Biosens. Bioelectron. 2000, 15, 453-462.

(18) Chen, W.-C.; Lee, L.-H.; Chen, B.-F.; Yen, C.-T. J. Mater. Chem. 2002, 12, 3644-3648.

(19) Yang, P.; Wirnsberger, G.; Huang, H. C.; Cordero, S. R.; McGehee, M. D.; Scott, B.; Deng, T.; Whitesides, G. M.; Chmelka, B. F.; Buratto, S. K.; Stucky, G. D. Science 2000, 21 (5452), 465-467.

(20) Coakley, K. M.; Liu, Y.; McGehee, M. D.; Frindell, K. L.; Stucky, G. D. Adv. Funct. Mater. 2003, 13 (4), 301-306.

(21) Couturier, C.; Leduc, P.; Farjot, T.; Passemard, G. Proc. Advanced Metallization Conf., 2006 2007, 395-399.

(22) Guyer, E. P.; Gantz, J.; Dauskardt, R. H. J. Mater. Res. 2007, 22 (3), 710-718.

(23) Shamiryan, D.; Maex, K. Mater. Res. Soc. Symp. Proc. 2003, 766, E9.11.1-6.

(24) Abell, T.; Shamiryan, D.; Patz, M.; Maex, K. Proc. Advanced Metallization Conf., 2003, 2004.

(25) Abell, T.; Schuhmacher, J.; Tokei, Z.; Travaly, Y.; Maex, K. Microelectron. Eng. 2005, 82, 411-415.

(26) Worsley, M. A.; Roberts, M.; Bent, S.; Gates, S. M.; Shaw, T.; Volksen, W.; Miller, R. D. Microelectron. Eng. 2005, 82, 113-118.

(27) Shamiryan, D.; Baklanov, M. R.; Lyons, P.; Beckx, S.; Boullart, W.; Maex, K. Colloids Surf., A 2007, 300, 111-116.

(28) Chang, H. C.; Hsu, C. T.; Lin, S. Y. Langmuir 1998, 14, 2476-2484.

(29) Lin, S. Y.; Tsay, R. Y.; Lin, L. W.; Chen, S. I. Langmuir 1996, 12, 6530-6536.

(30) Klein, J. Nature 1978, 271, 143-145.

(31) Hervet, H.; Léger, L.; Rondelez, F. Phys. Rev. Lett. 1979, 42, 16811684.

(32) Tanner, J. E.; Liu, K. J.; Anderson, J. E. Macromolecules 1971, 4 (5), 586-588.

(33) Bachus, R.; Kimmich, R. Polymer 1983, 24, 964-970.

(34) Richter, D.; Farago, B.; Fetters, L. J.; Huang, J. S.; Ewen, B.; Lartigue, C. Phys. Rev. Lett. 1990, 64 (12), 1389-1392.

(35) de Gennes, P. G., Scaling Concepts in Polymer Physics; Cornell University Press: Ithaca and London, 1979.

(36) Zheng, X.; Sauer, B.; Alsten, J. G. V.; Schwarz, S. A.; Rafailovich, M. H.; Sokolov, J.; Rubinstein, M. Phys. Rev. Lett. 1995, 74 (3), 407-410.

NL901138P 